\title{
Predicting response bounds for friction-damped gas turbine blades with uncertain friction coupling
}

\author{
T. Butlin ${ }^{\mathrm{a}, *}$, G. Spelman ${ }^{\mathrm{a}}$, P. Ghaderi ${ }^{\mathrm{a}}$, W. J. B. Midgley ${ }^{\mathrm{b}}$, \\ R. Umehara ${ }^{\mathrm{b}}$ \\ ${ }^{a}$ Cambridge University Engineering Department, Trumpington Street, Cambridge, \\ CB2 1PZ, England \\ ${ }^{\mathrm{b}}$ Vibration No.3 Laboratory, Vibration Research Department, Research and \\ Innovation Center, Mitsubishi Heavy Industries, Ltd., 2-1-1 Shinhama, Arai-cho, \\ Takasago City, Hyogo, 676-8686, Japan
}

\section{Highlights}

- A new method to predict response bounds is applied to friction-damped systems.

- The approach applies to parametric and model uncertainty associated with friction.

- Bounds can be computed at similar computational cost to a single HBM simulation.

- Results are compared with an eight-blade idealised laboratory test rig.

- Comparisons with numerical and experimental Monte Carlo tests show good agreement.

\begin{abstract}
Friction dampers are often used to reduce high amplitude vibration within gas turbines: they are a robust solution that are able to withstand extreme operating environments. Although the turbine blades are manufactured to tight tolerances, there can be significant variability in the overall response of the assembly. Uncertainties associated with the frictional contact properties are a major factor contributing to this variability. This paper applies a recently developed method for predicting response bounds to friction-damped gas turbines when the characteristics of the friction dampers are unknown, including uncertainty regarding the underlying functional form of the friction law. The approach taken is to represent the frictional contact using a describing function, and formulate an optimisation problem to seek upper and lower bounds on a chosen response metric, such as displacement amplitude. Constraints are chosen that describe known properties of the frictional nonlinearity, without needing to specify a particular constitutive law. The method was validated by comparison with numerical and experimental results from an idealised test system. The experimental test rig consisted of an array of eight beams
\end{abstract}


coupled by pin-contact friction dampers. A modal description of this test rig formed the basis of a numerical model, which uses the Harmonic Balance Method (HBM) for nonlinear simulations. A set of Monte Carlo tests was carried out numerically and experimentally for both a two-beam sub-assembly as well as for the full eightbeam assembly. Comparisons with numerical results showed excellent agreement providing confident verification of the implementation, and comparisons with experimental results revealed that the bounds became less conservative as the system complexity increased. Overall the results are promising: upper and lower response bounds for an array of friction-damped systems can be computed at similar cost to a single HBM simulation, giving reliable bounds that are valid for both parametric and model uncertainties associated with the friction couplings.

Key words: nonlinear vibration, uncertainty, localised nonlinearities, turbine blades, response bounds, friction damping, underplatform dampers

\section{Introduction}

Friction dampers are commonly used to reduce high-amplitude vibration in gas turbines, in part due to their robustness under harsh operating conditions. But while the main structural components of turbines are manufactured to tight tolerances, it is not possible to control all of the dynamic properties during operation, leading to uncertainty in the dynamic behaviour of the system. One of the major sources of uncertainty is the frictional contact properties, to the extent that the functional form of the frictional law itself is unknown [1]. Predicting the response distribution of nonlinear systems with uncertainty is challenging: many uncertainty propagation methods require multiple simulations of the nonlinear system which is often computationally expensive.

There is a need for efficient methods that can predict the response of frictiondamped structures and which take uncertainty into account, without requiring computationally demanding Monte Carlo simulations of the nonlinear system. There are two main strategies: develop computationally efficient modelling methods so that Monte Carlo studies become feasible (e.g. [2,3]); and / or develop methods for handling uncertainty that require a minimal number of nonlinear simulations (e.g. [4]).

There is a growing variety of methods for handling uncertainty in structural

\footnotetext{
* Corresponding author (tel: +44 1223 765237)

Email addresses: tb267@cam.ac.uk (T. Butlin), gms41@cam.ac.uk (G.

Spelman), pg452@cam.ac.uk (P. Ghaderi ), william_midgley@mhi.co.jp (W. J.

B. Midgley), ryuichi_umehara@mhi.co.jp (R. Umehara).
} 
dynamics and several helpful special issues have been published, e.g. [5-7]. For the purposes of this paper it is helpful to distinguish between methods that are applicable to parametric or non-parametric types of uncertainty (where 'parametric' here refers to model parameters). Parametric methods assume knowledge of the governing equations of the system, and identify parameters within the model that are unknown.

There are two challenges common to parametric methods: they require multiple simulations to be carried out to predict the response of the system for different choices of system parameters; and the functional form of the governing equations of the system needs to be specified. There are methods emerging that begin to tackle the first issue. For example, in the context of probabilistic uncertainties Peherstorfer et al. [4] use importance sampling together with a combination of surrogate models and high fidelity models to obtain an efficient estimate of the response statistics. That there is a need for this kind of multi-resolution algorithm itself highlights the difficulty, and there is still a need for multiple simulations of a high resolution model.

Fuzzy arithmetic is another method applicable to non-probabilistic types of uncertainty, but as described by Moens and Hanss [8] the efficiency of fuzzy arithmetic methods is still limited by the number of simulations needed to estimate response bounds for different levels of uncertainty membership. This is because the response bounds are found by optimisation, or for the upper bound by 'anti-optimisation': in other words numerical optimisation is used to search the admissible set of parameters for the extreme responses. For both of these example methods the governing system equations need to be prespecified as 'knowns' and parametric uncertainty methods intrinsically cannot account for 'model' uncertainty.

Another method that has started to received significant attention is the use of Polynomial Chaos Expansion (PCE): the fundamental theoretical work was developed in [9], but it has only more recently begun to be applied in engineering applications [10]. The core approach is to describe the uncertain input parameters and response distribution in terms of a truncated set of orthogonal basis distributions, then solve the system of equations for the coefficients of the output distribution basis. This can be achieved either by Galerkin projection (referred to as an 'intrusive' method in the sense of changing the system of equations to solve), or by least squares solution using point-wise observations from the original simulation code (referred to as a 'non-intrusive' method). The intrusive methods are computationally faster but more complex to implement [11]. This class of uncertainty propagation method can be very efficient and applicable to nonlinear systems: the approach has been combined with the multi-frequency harmonic balance method using both intrusive [12] and non-intrusive approaches [13], and also with a nonlinear normal mode framework [14]. The results in each study show a great deal of potential, accounting 
for uncertainty of strongly nonlinear systems with multi-stable frequency response curves. Nevertheless there are several underlying challenges associated with PCE methods in general: simulation time scales poorly with the number of uncertain parameters, currently limiting the complexity of system that can be tackled; and there are open questions about convergence and error quantification of the output expansion [10].

These shortcomings make non-parametric methods appealing, but their domain of applicability is more specific. For example Statistical Energy Analysis (SEA) enables efficient prediction of the mean and variance of the steady-state response for linear systems at high frequencies, i.e. when there is significant statistical overlap [15]. It remains a challenge to apply the concepts of SEA to nonlinear systems, though some interesting progress has been made recently $[16]$.

This paper presents a recently developed method [17-20] for estimating the upper and lower response bounds of friction-damped gas turbine blades, specifically for the case when there is uncertainty associated with the nonlinear friction interaction. The key features of the method are that:

- uncertainty is represented by specifying general properties of the nonlinearity, so the functional form of the friction law does not need to be specified;

- only the linear forced response needs to be computed to estimate the upper and lower bounds on the response;

- it is most efficient when the nonlinearities are spatially localised.

The method presented can be viewed as a parametric uncertainty approach: but its novelty is that the uncertain parameters are applied to the describing function of the nonlinearity, which avoids the need for Monte Carlo simulations of the nonlinear system. The advantages of this approach are: intrinsically including nonlinear model uncertainties; and being particularly efficient for complex systems with localised nonlinearities. The intrinsic disadvantage of response bounds methods in general is that they do not provide information about the response distribution: this is considered in a separate study.

This paper is structured as follows: Section 2 summarises a benchmark academic reference model that is based on an experimental test rig; Section 3 presents the details of the proposed method for estimating the bounds; and Section 4 presents a comparison of response bounds predictions with experimental and numerical Monte Carlo simulations from the benchmark model. 


\section{Benchmark reference model}

In order to validate the proposed approach, a simplified academic test system has been designed that retains the key features of friction-damped systems. The key design requirements of the system were that it should be periodic to represent the periodicity of bladed disks, and include frictional couplings between periodic elements. With this starting point, an experimental test rig was designed that consists of a periodic array of beams coupled by friction dampers. The purpose of the reference model was to enable experimental and numerical Monte Carlo tests: generating ensembles of data with controlled uncertainty that could then be compared with upper and lower bound predictions.

\subsection{Overall design}

Figure 1 shows a photograph of the eight-beam experimental test rig, where the beams will be referred to as Beams 1-8 from left to right. Figure 2 shows an annotated diagram for (a) a nominal beam and (b) a friction coupling arm. Note that the array is non-circular as this considerably simplifies the design and manufacture of the rig: circular periodicity is not a fundamental requirement for validating the response bounds approach. The beams have been water-jet cut from a single sheet of steel to form a comb-like structure of eight nominally identical beams connected at their base for straightforward alignment. The base is bolted between two heavy clamping beams (each $20 \mathrm{~mm}$ thick) to minimise coupling between the beams via the base. The top of each beam is folded over to provide a horizontal surface for sliding contact when the beams vibrate out-of-plane. Each beam is $300 \times 40 \times 3 \mathrm{~mm}$ (height $\times$ width $\times$ thickness), and the length of the folded over section of the beam is $31 \mathrm{~mm}$.

Each beam can be independently excited by a non-contact coil-magnet arrangement at Position 3 (see Fig. 2 for Position labels). This consists of a neodymium cylinder magnet (diameter $10 \mathrm{~mm}$, length $20 \mathrm{~mm}$ ) attached to the beam, positioned within a coil $(120 \mathrm{~m}, 21 \mathrm{awg})$ that is clamped to ground. Accelerometers (DJB A/20) are used to measure the response on each beam at approximately the mid-point (Position 2) and near the top (Position 1) of the beams.

Friction coupling is introduced by an aluminium arm that connects the tops of each pair of beams: the arm has a thin flexure that allows a known vertical preload to be applied using weights suspended on a soft spring, while still retaining bending stiffness in the other directions. A hemispherical pin is mounted at the tip of each coupling arm, which comes into frictional contact with the 
horizontal platform at the top of the adjacent beam. In order to maintain approximately constant normal force during large amplitude oscillations, a small steel wedge was secured onto the beam platform to provide a frictional contacting surface at an angle of approximately 3 degrees.

In addition, each beam has a moveable brass mass (approximately $70 \mathrm{~g}$ ) to allow controlled mistuning of the test rig: this paper focusses on the specific case of uncertainty associated with the frictional nonlinearity, so the model used here is based on the tuned configuration with all masses at the same position (mid-point).

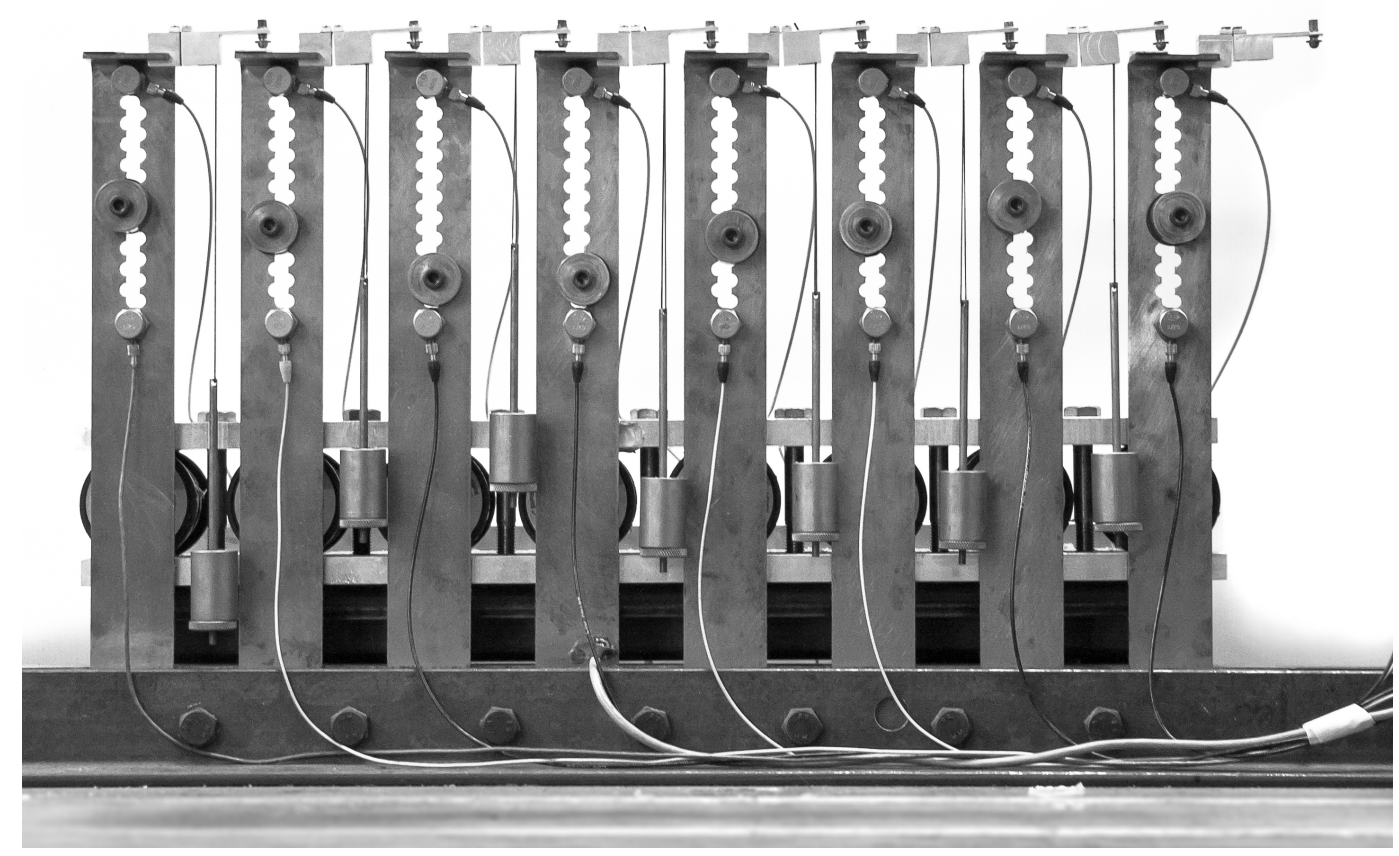

Fig. 1. Photograph of the experimental test rig that forms the basis of the benchmark reference model. Beams are numbered 1-8 from left to right.

\subsection{Harmonic Balance Method implementation}

The Harmonic Balance Method (HBM) is commonly used for predicting the response of friction damped turbine blades, e.g. [21]. The solution is approximated as a truncated series of harmonic terms with fundamental frequency usually chosen to be the input forcing frequency, and the numerical precision of predictions increases with the number of terms included in the expansion [22]. This is a robust and well-understood method, and it has been found that satisfactory (albeit more approximate) predictions can be achieved even when only the fundamental frequency is retained in the expansion [23]. This apparently severe assumption still gives useful results because for friction damped sys- 


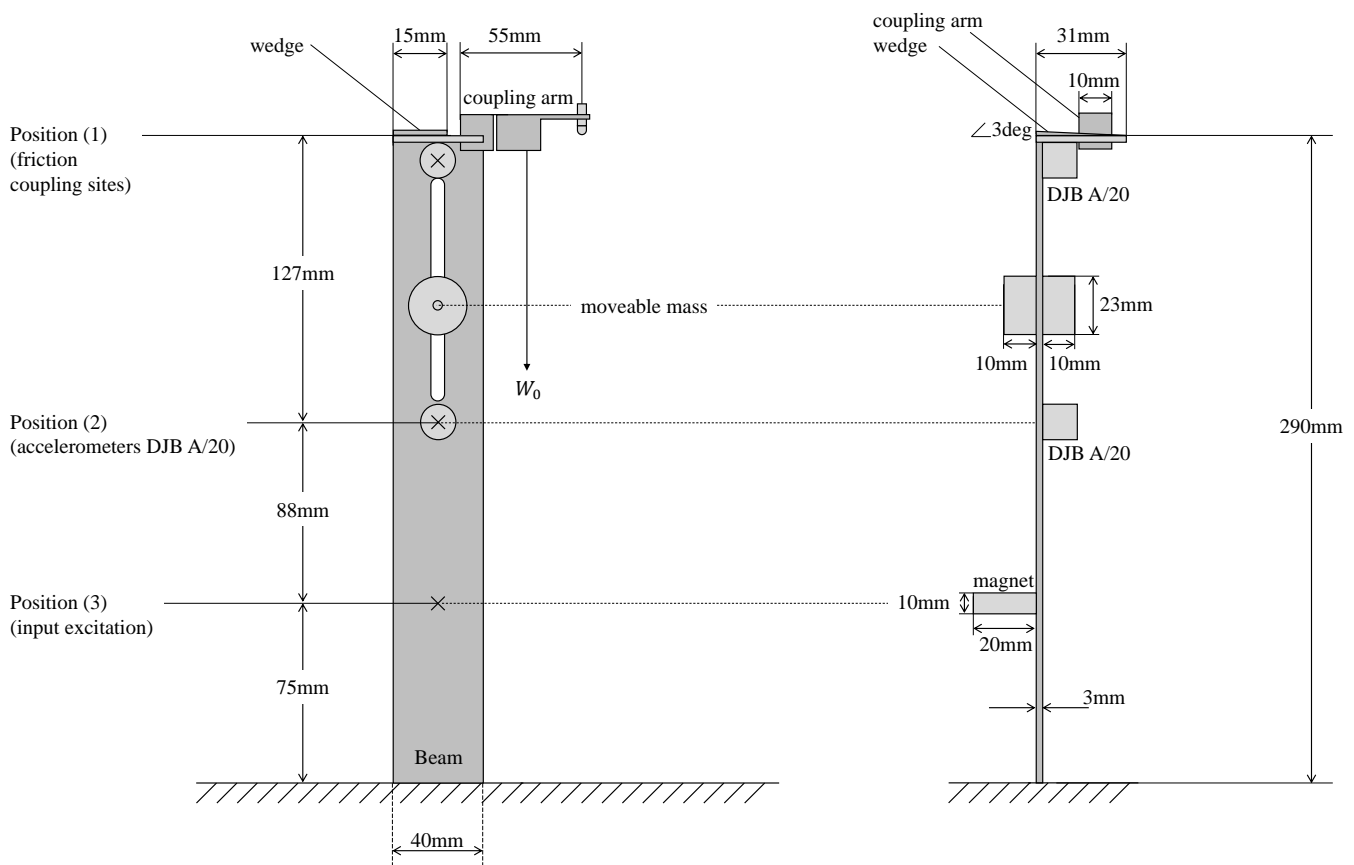

(a)

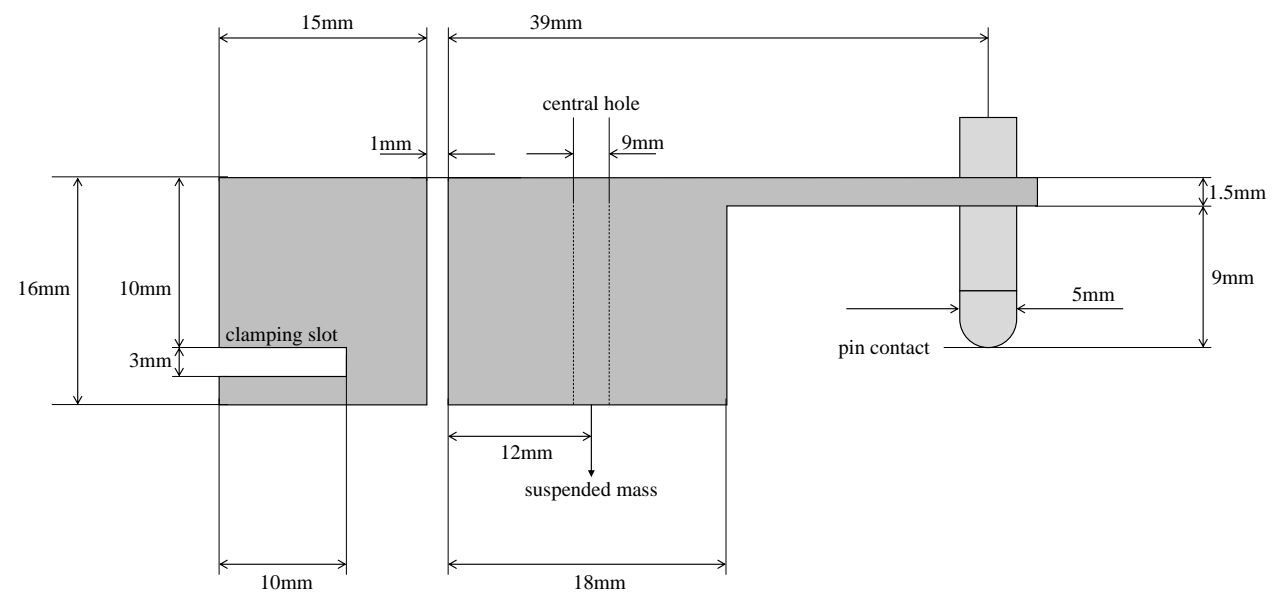

(b)

Fig. 2. Diagram showing dimensions (not to scale) of (a) a single beam within the array and (b) details of a friction coupling arm. 
tems it is usually the case that the output displacement response is dominated by the fundamental excitation frequency. The approach represents a form of linearisation and the nonlinear system is characterised by an amplitude and frequency dependent 'describing function'. This first-order approach is used in the present study as the intention is to focus on the effects of uncertainty rather than to achieve high accuracy deterministic predictions.

The frequency-domain representation of the friction-damped system is summarised in Fig. 3. The total force $\mathbf{F}$ acting on a set of beams is the sum of external forces $\mathbf{F}_{\text {ext }}$ and internal nonlinear friction forces $\mathbf{F}_{\mathrm{nl}}$. The linear structural dynamics can be characterised by the Frequency Response Function (FRF) matrix $\mathbf{D}(\omega)$ such that the output response is given by $\mathbf{Y}=\mathbf{D F}$. A subset of these output states $\mathbf{Y}_{\mathrm{nl}}$ are associated with the nonlinear friction dampers, and these states provide the input to the friction describing function $\mathbf{K}\left(\omega, \mathbf{Y}_{\mathrm{nl}}\right)$. An output metric is defined by a mapping $M$, and is simply an output quantity of interest chosen by the user. The feedback representation might suggest the possibility of instability and self-excited vibration: while friction contacts can and do lead to self-excited vibration (e.g. [24]) this is not normally considered to be an issue in the context of friction dampers as the mean sliding velocity is zero, and the possibility of squeak during reciprocating sliding is beyond the scope of this study.

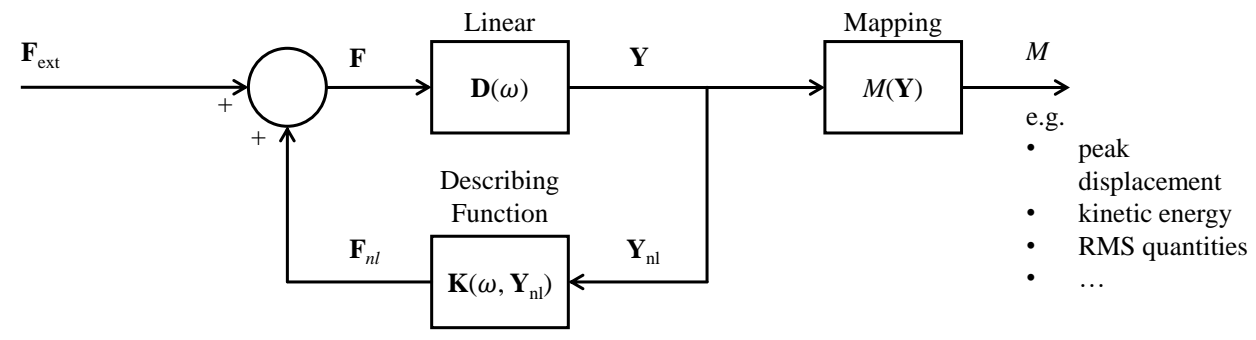

Fig. 3. Summary of friction-damped system representation using a describing function $\mathbf{K}$ to characterise the behaviour of friction dampers.

A given element of the linear FRF matrix $\mathbf{D}$ can be written:

$$
D_{n, m}(\omega)=\sum_{\text {all } k} \frac{u_{n}^{(k)} u_{m}^{(k)}}{\omega_{k}^{2}+2 \mathrm{i} \zeta_{k} \omega_{k} \omega-\omega^{2}}
$$

where $u_{n}^{(k)}$ is the modal amplitude at Position $n, \omega_{k}$ is the natural frequency and $\zeta_{k}$ is the modal damping factor, all for the $k$ th mode. Experimental modal analysis was carried out to obtain the modal properties of each beam using standard procedures (e.g. [25]): the properties of the first two modes are sum- 
marised in Table 1. These two modes represented the simplest behaviour of the test rig where the mode shapes were predominantly out-of-plane bending modes. Torsional and higher order bending modes were identified above $150 \mathrm{~Hz}$ : for simplicity the bandwidth of interest was chosen to be $0-150 \mathrm{~Hz}$.

Table 1

Nominal modal properties for the first two modes of a single beam, as identified from measurements of Beam 6 .

\begin{tabular}{ccccccc}
\hline Mode $k$ & Type & Frequency $(\mathrm{Hz})$ & Damping & $u_{1}^{(k)}$ & $u_{2}^{(k)}$ & $u_{3}^{(k)}$ \\
\hline 1 & Bending & 16.20 & 0.0017 & 2.55 & 0.99 & 0.48 \\
2 & Bending & 105.8 & 0.0071 & -1.84 & 2.18 & 1.42 \\
\hline
\end{tabular}

Fig. 4 shows an example comparison of a measured transfer function for Beam 6 with its modal reconstruction, using an input impulsive excitation at Position (2) (mid-point of the beam) and measuring the output acceleration response at Position (1) in the frequency range $0-150 \mathrm{~Hz}$. It can be seen that the two resonant peaks are accurately represented, noting that the low frequency experimental data is less reliable (due to high-pass filters on input and output charge amplifiers). The small differences near $150 \mathrm{~Hz}$ are due to resonant peaks that fall outside this bandwidth that have not been fitted. Therefore in this frequency range the linear structural dynamics in the absence of friction dampers can be represented deterministically using Eq. (1).

The simplest contact model has been chosen and is based on [23], which uses a describing function to represent the nonlinear friction dampers. The underlying friction law was assumed to be Coulomb's law in series with a tangential stiffness. The tangential stiffness $k_{c}$ usually corresponds to the contact stiffness at the interface, but for our test rig the stiffness of the friction arm was much lower than the local contact stiffness and so was the dominant effect. However, this makes no mathematical difference to the contact law and only means that the stiffness values are lower than might otherwise have been expected. The coefficient of friction for the steel-on-steel contact was measured using steady state measurements from a separate pin-on-disc tribometer: for full details of the tribometer see [26]. The combined coupling and contact stiffness was inferred by isolating each adjacent pair of beams (disconnecting and damping all other beams), applying a large contact pre-load so that the beams were close to the linear sticking-limit, then measuring the coupled transfer function. The frequency separation of the first two modes is governed by the coupling stiffness allowing this to be inferred.

Both contact parameters were highly variable: the coefficient of friction was measured to be in the range $0.5<\mu_{0}<0.8$, where the high values tended to correlate to low sliding speeds. The combined contact and coupling stiffness was found to be in the range $4.8<k_{c}<8.0 \mathrm{kNm}^{-1}$ : this is lower than might be expected for contact stiffness alone because $k_{c}$ here is the combined stiffness 


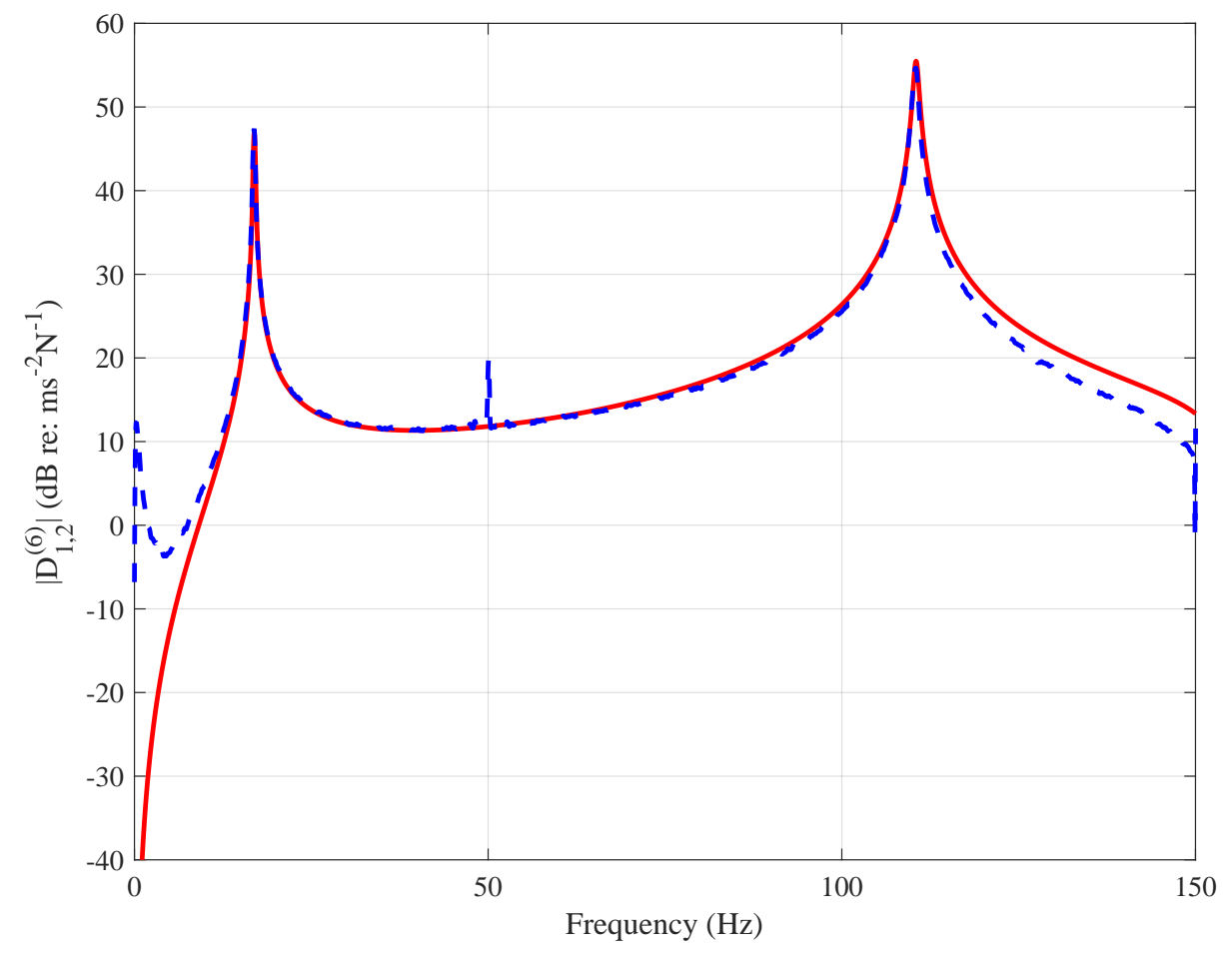

Fig. 4. Magnitude of transfer function $D_{1,2}^{(6)}$ for beam 6 in isolation (no friction coupling to other beams) from input force at Position (2) to output acceleration near the tip at Position (1). Dashed line: measured response; Solid line: modal reconstruction.

of the coupling arm in series with the frictional contact stiffness.

It is convenient to define a non-dimensional parameter $s$ :

$$
s \equiv \frac{\mu_{0} N_{0}}{k_{c} A} .
$$

where $\mu_{0}$ is the coefficient of friction, $N_{0}$ is the normal contact pre-load, $k_{c}$ is the coupling stiffness, and $A$ is the relative displacement amplitude at the friction contact. The describing function $K$ for a single damper can be written as a function of $s$ :

$$
K=K_{r}(s)+\mathrm{i} K_{i}(s),
$$

where

$$
\begin{aligned}
K_{r} & =\frac{k_{c}}{\pi}(\arccos (1-2 s)-2(1-2 s) \sqrt{s(1-s)}) \\
K_{i} & =\frac{4 k_{c}}{\pi} s(1-s),
\end{aligned}
$$

which is valid for $0<s \leq 1$. When $s \rightarrow 0$ then $K \rightarrow 0$ which corresponds to 
large relative amplitude (the fully slipping limit), and when $s=1$ then $K=k_{c}$ which corresponds to small relative amplitude (the fully sticking limit). These results were derived in [23], and the expressions presented here use notation consistent with the present study.

Eq. (3) represents the describing function for a single damper, so the response of $N$ dampers is parameterised by the vector $\mathbf{s}=\left[\begin{array}{llll}s_{1} & s_{2} & \cdots & s_{N}\end{array}\right]^{T}$ corresponding to the diagonal matrix of describing functions $\mathbf{K}$ (see Fig. 3). The solution procedure adopted was to:

(1) guess a vector of values $\mathbf{s}_{\text {trial }}$

(2) compute the corresponding diagonal describing function matrix $\mathbf{K}$ using Eq. (3)-(5):

$$
\mathbf{K}=\left[\begin{array}{cccc}
K_{1} & 0 & \cdots & 0 \\
0 & K_{2} & & 0 \\
\vdots & & \ddots & \vdots \\
0 & 0 & \cdots & K_{N}
\end{array}\right]
$$

(3) find the response $\mathbf{Y}=(\mathbf{I}-\mathbf{D K})^{-1} \mathbf{D F}_{\text {ext }}$

(4) compute $\mathbf{s}_{\text {out }}$, with $s_{\text {out }, j}=\mu_{0, j} N_{0, j} / k_{c, j} A_{j}$, and $A_{j}$ being the relative displacement amplitude across the $j$ th friction damper computed from $\mathrm{Y}$

(5) iterate until $\mathbf{s}_{\text {trial }}-\mathbf{s}_{\text {out }}=0$.

The numerical solution was found using Matlab's fsolve function.

\subsection{Validation of HBM with experimental results}

The linear structural dynamics are well understood and accurately characterised over the bandwidth of interest of $0-150 \mathrm{~Hz}$. Introducing the frictional coupling makes predictions much more challenging due to both nonlinearity and uncertainty. As a starting point, two beams (5 and 6) were effectively isolated from the rest of the assembly, rather than starting with the full eightbeam friction-coupled test rig. To isolate this pair of beams, all the friction dampers were disconnected except for the one connecting beams 5 and 6 . This effectively decoupled the other beams because the base was tightly clamped by a thick steel beam. However, some small residual coupling was observed (due to finite stiffness of the clamping structure), so damping was added using foam inserts to further reduce the effect of the other beams. This was found empirically to be sufficient to observe the expected behaviour for a two-beam system. 
A sinusoidal input force excitation was applied to Beam 5 at Position (3) and the output acceleration response was measured at Position (2). A slow continuous frequency sweep was used for the input spanning 10-150 Hz. Convergence checks on the rate of change of frequency were carried out to ensure that unwanted transient effects were not significant (not shown). Figure 5 shows a comparison between HBM predictions and experimental results for (a) input excitation amplitude $F_{0}=0.18 \mathrm{~N}$ and (b) $F_{0}=0.72 \mathrm{~N}$. The dashed line shows the experimental results and the solid line is the HBM prediction, showing the response for the driven beam. The coefficient of friction found to give a qualitatively good fit across the amplitudes tested was $\mu_{0}=0.5$. It can be seen that overall there is reasonable agreement, with very good agreement in the range $0-100 \mathrm{~Hz}$. This range includes the first two peaks at $17 \mathrm{~Hz}$ (beams in-phase) and $40 \mathrm{~Hz}$ (beams out-of-phase) corresponding to the first bending mode of the beams, i.e. the first passband of the coupled system. As expected the friction damper does not affect the in-phase mode $(17 \mathrm{~Hz})$ where there is theoretically no relative motion between the beams, but it has an increasing affect on the out-of-phase mode $(40 \mathrm{~Hz})$ where there is significant relative motion between the beams.

The level of agreement is more approximate in the second passband in the range 100-150 Hz: the two peaks again correspond to the in- and out-of-phase modes, in this case for the second bending mode of the beams. The reason for the larger discrepancy here is likely to be because the second modes of the beams are not tuned as accurately as the first modes of the beams (due to manufacturing and assembly details), while the HBM model assumes identical beams for these tests. These are details that could all be characterised in more detail, but the emphasis of this study is on predicting bounds arising from uncertainty associated with the friction and contact properties, rather than high fidelity modelling of the deterministic components, so correction of these details has not been carried out in this study.

A similar comparison was carried out for the full eight-beam assembly. In this case the excitation was applied to all eight-beams (rather than just one) in a pattern corresponding to the experimentally identified third passband mode of the assembly: this approximately corresponds to Engine Order 2 excitation, which has two nodal diameters and theoretically should only excite the corresponding passband mode for a perfectly tuned assembly.

Figure 6 shows the eight-beam comparison between HBM predictions and experimental results. In order to simplify the figure the maximum response across the eight beams is shown at any given frequency. This is consistent with the metric chosen for the response bounds that will be presented in Section 3. It can be seen that the results are qualitatively in broad agreement: peaks are at approximately the correct amplitudes, and the passband modes are more lightly damped for the lower excitation in (a) than in (b). However, 


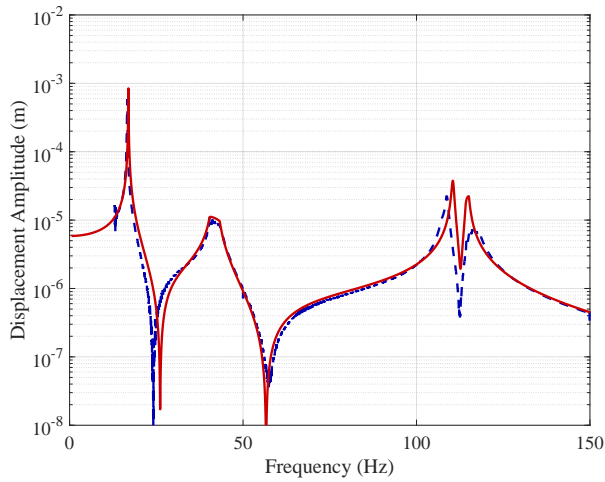

(a)

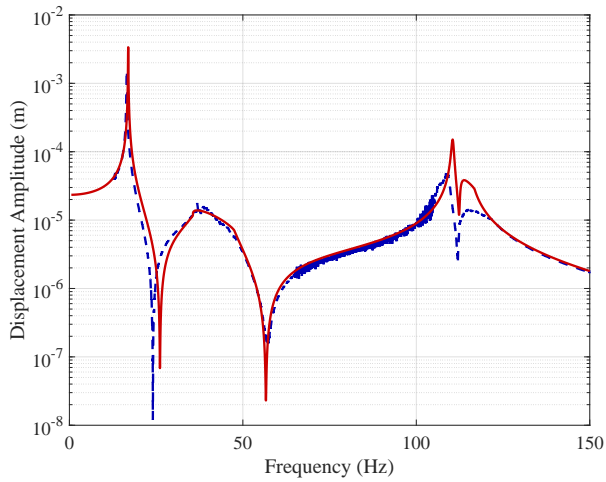

(b)

Fig. 5. Two-beam sub-assembly: comparison of HBM predictions (solid) with experimental swept-sine tests (dashed): (a) input force amplitude $F_{0}=0.18 \mathrm{~N}$; and (b) $F_{0}=0.72 \mathrm{~N}$.

there are significant differences in the details: this is wholly representative of how difficult it can be to obtain good agreement for assembled structures with frictional interfaces, and motivates the need for an approach that incorporates uncertainty intrinsically.

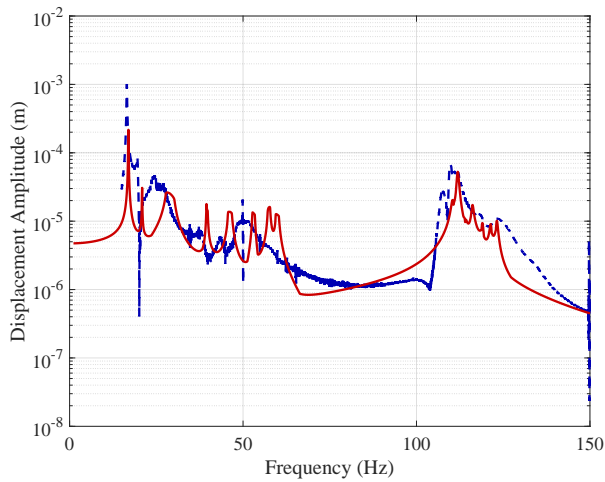

(a)

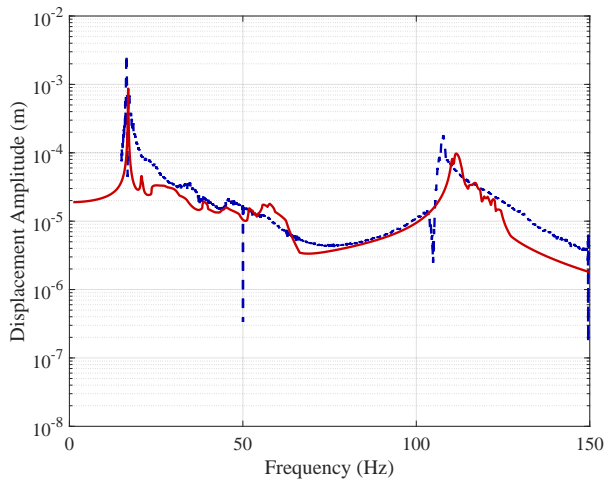

(b)

Fig. 6. Eight-beam full assembly: comparison of HBM predictions (solid lines) with experimental swept-sine tests (dashed): (a) input force amplitude $F_{0}=0.18 \mathrm{~N}$; and (b) $F_{0}=0.72 \mathrm{~N}$.

\section{Equivalent linear bounds framework}

The general approach for estimating the response bounds can be summarised as follows:

(1) Consider the nonlinearity to be an external excitation force; 
(2) Define constraints that describe general properties of the nonlinear friction interaction law;

(3) Find the nonlinear force that minimises or maximises an output quantity of interest subject to the constraints.

This approach can be formulated as an optimisation problem, the key advantage over other methods being that it only requires the linear forced response to be computed when seeking the response bounds. The optimisation problem can be solved using standard numerical optimisation toolboxes (e.g. Matlab's fmincon algorithms) $[17,18]$ and in some cases semi-analytic solutions can be found $[20]$.

In the general case, the solution gives a force that may have several frequency components, i.e. the worst case response occurs when there is a transfer of energy from the driving frequency to another frequency or combination of frequencies [20]. However, it is sometimes the case that the output response metric of interest is dominated by the contribution at the driving frequency: in this case the nonlinear force can be constrained to only have a component at the excitation frequency. This effectively means finding 'equivalent linear bounds' on the response. It may seem a severe restriction that is only applicable to very weakly nonlinear systems, however it has the same basis as the describing function approach presented above. The results above show that this can be effective even for systems with discontinuous nonlinearities and it has found widespread adoption for predicting the response of friction-damped systems.

The framework presented in this paper is based on the frequency-domain HBM system representation shown in Fig. 3: the key difference is that high-level constraints are chosen that define an admissible region of the describing function, and an output response metric is selected for which bounds are sought. The implicit effect of this is that the method does not require specification of the friction law, so it can account for a very broad class of uncertainty.

\subsection{General optimisation problem}

The equivalent linear bounds approach can written as a standard optimisation problem:

$$
\begin{aligned}
\underset{\mathbf{K}}{\operatorname{maximise}} & M\left(\mathbf{K}, \mathbf{F}_{\mathrm{e} x t}\right) \\
\text { subject to: } & \mathbf{h}\left(\mathbf{K}, \mathbf{F}_{\mathrm{e} x t}\right) \leq \mathbf{0},
\end{aligned}
$$

where $M$ is the response metric, $\mathbf{K}$ is the vector of describing functions for each damper, and $\mathbf{h}$ is the vector of inequality constraint functions. The input excitation force $\mathbf{F}_{\mathrm{e} x t}$ is assumed to be known. The maximum and minimum 
response metrics $M$ are sought by varying the describing function $K$ : the degrees of freedom for the optimisation are taken to be the real and imaginary parts of $K$ for each damper: therefore the computational cost for obtaining response bounds is similar to computing a single HBM simulation for a specific set of parameters.

The constraints $\mathbf{h}$ define the admissible search space for the describing function $K$, and the particular choice of constraints depends on what is assumed to be known about the nonlinear friction dampers. As more information is known, more constraints can be imposed and the predicted bound becomes less conservative (as demonstrated in [17]). Some choices of constraints for particular applications can lead to discontinuous constraint functions that require numerically challenging optimisation to find solutions (e.g. [18]). The aim of this paper is to select bounds with a physical basis that also allow solutions to be readily computed. With this in mind, the constraint functions identified for each damper are as follows:

$$
\begin{array}{ll}
h_{A}=-\operatorname{Re}\{K\} & \text { (positive stiffness) } \\
h_{B}=-\operatorname{Im}\{K\} & \text { (dissipative) } \\
h_{C}=\operatorname{Re}\{K\}-k_{\max } & \text { (maximum stiffness) } \\
h_{D}=\operatorname{Im}\{K\}-k_{\max } & \text { (maximum dissipation) } \\
h_{E}=F_{\mathrm{nl}}-F_{\lim } & \text { (friction force saturation) }
\end{array}
$$

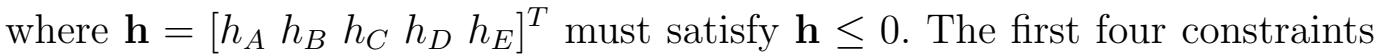
$h_{A}$ to $h_{D}$ define a bounding box on the describing function $K$ of each damper. The last constraint $h_{E}$ defines a force saturation limit associated with friction: simpler and clearer to write as a function of $F_{n l}$ rather than $K$. These constraint functions are not unique, and are chosen here to demonstrate the general approach.

\subsection{Solution method}

The solution to this optimisation problem is computed using Matlab's fmincon toolbox using the sqp algorithm. However a direct approach is inefficient: the admissible region of the describing function $K$ defined by the constraints in Eq. (8) is non-trivial due to the force constraint $h_{E}$. To improve convergence and scalability, the solution is computed as follows:

(1) Compute the objective function using a set of values of $K$ on the boundaries defined by $h_{A}$ to $h_{D}$, choosing uncorrelated values across the set of dampers; 
(2) Use the smallest objective as an initial guess for optimisation using fmincon, accounting only for the constraints $h_{A}$ to $h_{D}$;

(3) analytically find the minimum and maximum response due to the force constraint on its own;

(4) identify the limiting bounds.

The upper bound due to the force constraint on its own is found by assuming all the friction dampers are acting at their force limits, and that their phase causes the largest magnitude response. The output response is given by:

$$
\mathbf{Y}=\mathbf{D}\left(\mathbf{F}_{\mathrm{ext}}+\mathbf{F}_{\mathrm{nl}}\right)
$$

giving an upper bound:

$$
|\mathbf{Y}| \leq\left|\mathbf{D F}_{\text {ext }}\right|+F_{\text {lim }}|\mathbf{D}| \hat{\mathbf{F}}_{\mathrm{nl}}
$$

where $\hat{\mathbf{F}}_{\mathrm{nl}}$ is a binary vector identifying the friction-contacts. The lower bound is found just using the constraints $h_{A}$ to $h_{D}$ as the force constraint is less important for this bound.

\section{Results and comparisons}

Results will be presented for the two-beam sub-assembly before showing the comparison with the full eight-beam system.

\subsection{Two-beam comparisons}

The method described in Section 3 for predicting response bounds is based on uncertainty associated with the properties of the frictional coupling. Therefore, in order to test the effectiveness of the method, a Monte Carlo test was carried out using the reference model to generate an ensemble of responses. The friction law parameters were varied as follows: $0<\mu_{0}<1$ and $0<k_{c}<k_{\max }$. A uniform distribution was chosen for both parameters, choosing $k_{\max }=15 \mathrm{kNm}^{-1}$ (the measured range for the test rig was approximately 5 to $10 \mathrm{kNm}^{-1}$ ).

Figure 7 shows a comparison between the predicted bounds (bold lines) and Monte Carlo HBM results (grey cloud) using an ensemble of approximately 200 simulations, for (a) $F_{0}=0.22 \mathrm{~N}$ and (b) $F_{0}=1.1 \mathrm{~N}$. A single example simulation within the ensemble is shown as a solid black line: this ensemble member has no special significance and is only highlighted to show the typical structure of a single simulation. It is clear that the simulated data falls exactly 
within the predicted bounds, confirming that the bounds represent converged and reliable solutions to the optimisation problem defined by Eq. (3.1). It is interesting that the Coulomb-specific parameters $k_{c}$ and $\mu_{0}$ provide sufficient uncertainty that the bounds are exact, given that the uncertainty specification in terms of the describing function allows for other kinds of friction laws.

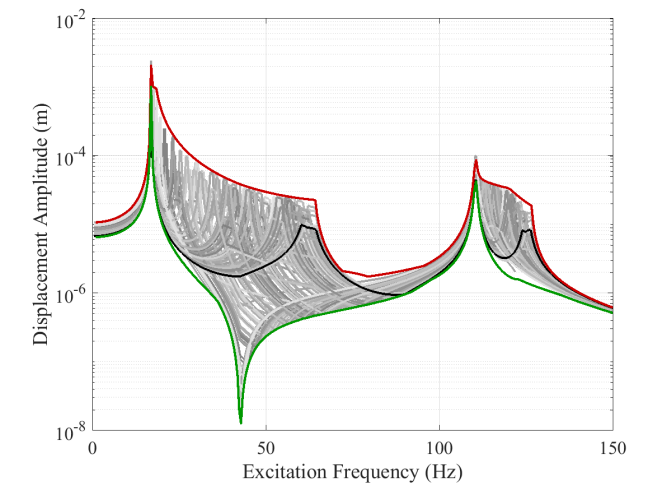

(a)

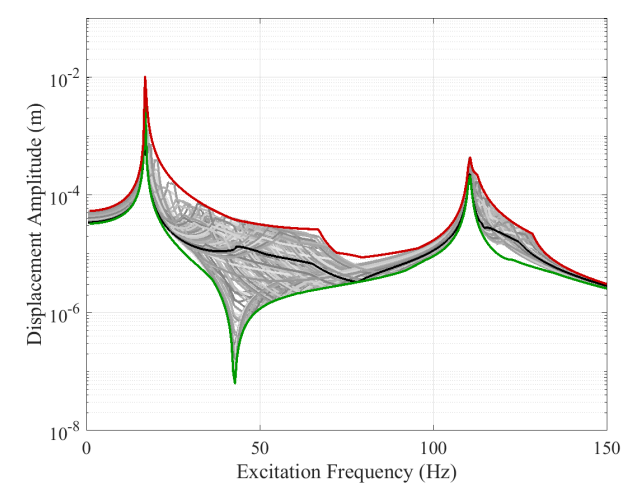

(b)

Fig. 7. Two-beam sub-assembly: comparison of HBM predictions (solid lines) with an ensemble of HBM simulations (grey lines): (a) input force amplitude $F_{0}=0.22 \mathrm{~N}$; and (b) $F_{0}=1.1 \mathrm{~N}$.

A similar experimental Monte Carlo test was also carried out. However, there is less flexibility to deliberately introduce uncertainty. In order to systematically vary the friction force limit, the normal preload was varied in the range $0.1<$ $N_{0}<0.75 \mathrm{~N}$. This resulted in an ensemble of three datasets. The experiments were carried out at an early stage of the project, and the frequency range under consideration (approximately 10-80 Hz) included just the first passband. The tests were carried out using a stepped-sine force input: a sinusoidal input was applied to beam 5 at Position (3); then the steady-state response amplitude at the driving frequency was measured.

Figure 8 shows the comparison of these experimental results (crosses) with the predicted bounds (solid lines) for (a) $F_{0}=0.22 \mathrm{~N}$ and (b) $F_{0}=1.1 \mathrm{~N}$. The experimental data is shown as crosses to denote stepped-sine tests, deliberately distinct from the lines corresponding to sine-sweep tests in other figures. It is reassuring that nearly all of the experimental results fall within the bounds, and that the data meets the bounds at some frequency ranges. The key underlying physics is again apparent. At low amplitudes it can be seen in (a) that some of the data reveals a truncated resonant peak near $55 \mathrm{~Hz}$ : this corresponds to the friction damper in a predominantly sticking state, and the peak is consistent with the coupled out-of-phase mode that would be expected from linear theory. The passband width is evident from the 'corner' in the upper bound plot near $65 \mathrm{~Hz}$, which is sensitive to the coupling stiffness bound. It is also interesting to see how the bounds become tighter at high amplitude as seen in (b), as the friction dampers tend towards the slipping limit. Both 
bounds are extremely conservative over the range $30-70 \mathrm{~Hz}$ and span approximately two orders of magnitude. This is because the constraints allow the effective coupling stiffness $k_{c}$ to fall within the range $0<k_{c}<15 \mathrm{kNm}^{-1}$. The worst-case occurs when it causes the resonance frequency of the out-of-phase coupled beam mode to be the same as the input frequency. As revealed by the numerical Monte Carlo study, if more experiments had been carried out using a wider range of contact stiffness values then this would have 'filled in the gap'.

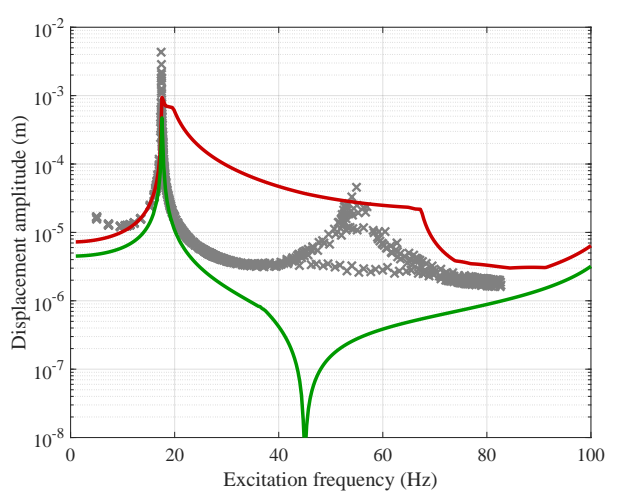

(a)

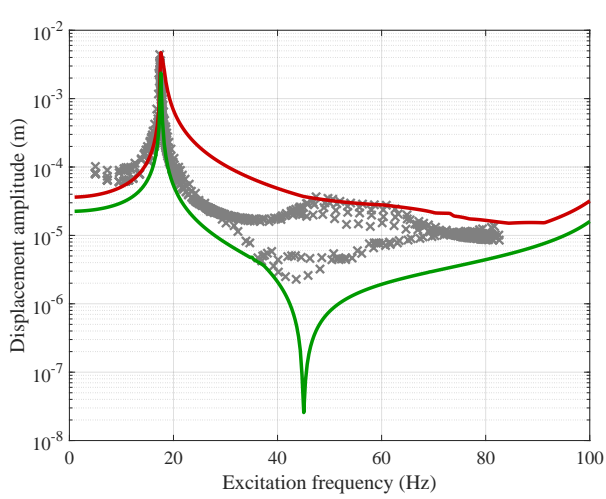

(b)

Fig. 8. Two-beam sub-assembly: comparison of HBM predictions (solid lines) with experimental stepped-sine tests (crosses): (a) input force amplitude $F_{0}=0.22 \mathrm{~N}$; and (b) $F_{0}=1.1 \mathrm{~N}$.

\subsection{Eight-beam comparisons}

The two-beam sub-assembly represents a highly idealised test case, so in order to begin testing the method on more complex structures, the response bounds were compared with Monte Carlo numerical and experimental tests using the full eight-beam assembly.

Figure 9 shows a comparison of the bounds with an ensemble of 200 Monte Carlo HBM simulations, equivalent to the two-beam comparison shown in Fig. 7 , in this case for (a) $F_{0}=0.25 \mathrm{~N}$ and (b) $F_{0}=1.1 \mathrm{~N}$ (values chosen to correspond to the eight-beam experimental data). The results again provide a clear verification that the optimisation algorithm is providing converged and reliable solutions for this more complicated system.

It is interesting that the bounds for the 2- and 8-beam cases (Figures 7 and 9) are rather similar. Note that the individual ensemble responses are significantly different as can be seen from the highlighted example responses in each figure. The similarity arises as a property of periodic structures where the passband is governed by the coupling strength, which is similar for the 2- and 8-beam 
cases.

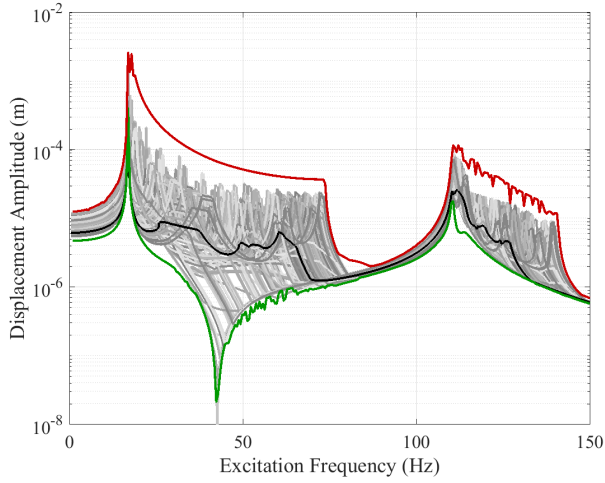

(a)

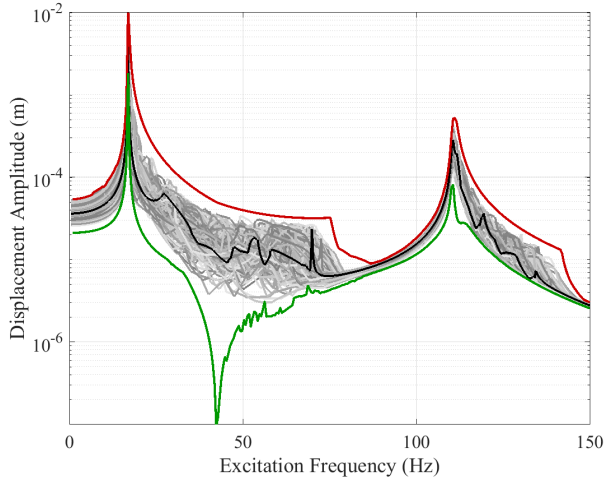

(b)

Fig. 9. Eight-beam full assembly: comparison of predicted response bounds (solid lines) with an ensemble of HBM simulations (grey lines): (a) input force amplitude $F_{0}=0.22 \mathrm{~N}$; and (b) $F_{0}=1.1 \mathrm{~N}$.

Conducting experimental Monte Carlo tests for the eight-beam rig is very labour intensive, requiring manual changes to the normal pre-load of each damper. However, with suitable normalisation the excitation amplitude can be used as a proxy for normal pre-load changes, and this can be varied automatically. This does not allow independent variations of normal pre-load but scaling the whole vector of inputs together simulates a uniform scaling of the pre-load. The output response $\mathbf{Y}$ to an actual excitation force $\mathbf{F}_{\text {ext }}$ is given by:

$$
\mathbf{Y}=\mathbf{D}\left(\mathbf{F}_{\mathrm{ext}}+\mathbf{F}_{\mathrm{nl}}\right)
$$

A simulated response $\mathbf{Y}_{\text {sim }}$ can be generated from an assumed simulation input force $\mathbf{F}_{\text {sim }}=c \mathbf{F}_{\text {ext }}$ where $c$ is a scaling factor:

$$
\mathbf{Y}_{\text {sim }}=c \mathbf{Y}=\mathbf{D}\left(\mathbf{F}_{\text {sim }}+c \mathbf{F}_{\mathrm{nl}}\right)
$$

Figure 10(a) shows a comparison of the eight-beam results with the corresponding bounds. The bounds encompass nearly all of the data, and are not overly conservative. It is interesting that with more modes in the passband the results approach the upper bound over a wider frequency range than for the two-beam case: this is because there are so many more modes in the passband distributed across this range, and due to nonlinearity and mistuning they are all excited even using approximately an EO2 excitation pattern. One interesting discrepancy is that the frequency of the second passband near $110 \mathrm{~Hz}$ appears to be lower than predicted. This is very likely due to the rig having changed over time since its initial characterisation. It also appears that the passband width is wider than given by the $k_{\max }=15 \mathrm{kNm}^{-1}$ limit. In fact, measurement of all friction couplings shows that the coupling stiffnesses vary in the range $4.8<k_{c}<8 \mathrm{kNm}^{-1}$. Figure 10 (b) shows the results if an ad-hoc correction is made for these factors, reducing the upper bound on coupling 
stiffness to $k_{\max }=10 \mathrm{kNm}^{-1}$ and adjusting the second beam mode frequency to $108 \mathrm{~Hz}$ from $110 \mathrm{~Hz}$. It can be seen that these changes further improve the agreement between the experimental results and the bounds.

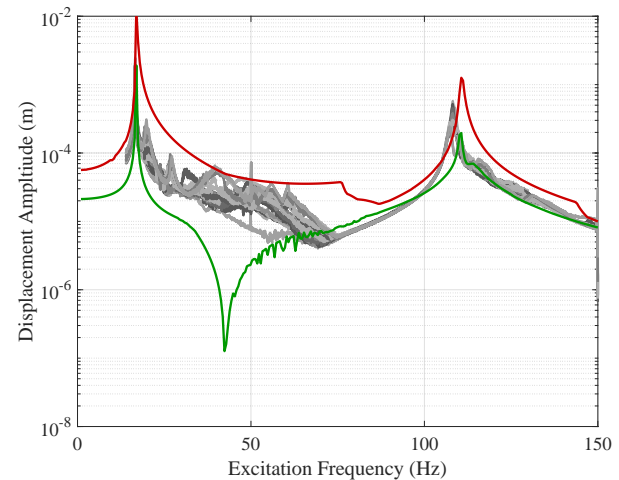

(a)

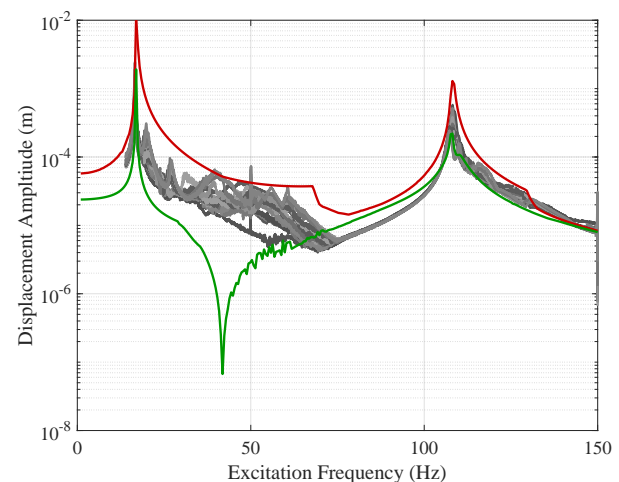

(b)

Fig. 10. Eight-beam full assembly: comparison of predicted response bounds (solid lines) with experimental sine-sweep tests (grey lines) with a simulated input force amplitude $F_{0}=1.1 \mathrm{~N}$ : (a) $0<k_{c}<15 \mathrm{kNm}^{-1}$; and (b) $0<k_{c}<10 \mathrm{kNm}^{-1}$, with adjusted second beam frequency to $f_{2}=108 \mathrm{~Hz}$.

\section{Conclusions}

There is a need for numerical methods that can efficiently predict the response variability of friction-damped turbine blades in the presence of uncertainty, without requiring computationally demanding Monte Carlo simulations of the nonlinear system. Although the turbine blades themselves are manufactured to very tight tolerances, there can be significant uncertainty associated with the frictional couplings arising for example from underplatform dampers.

This paper presents a novel approach to finding frequency-domain response bounds for sinusoidally excited turbine blades coupled by friction dampers for the case when there is uncertainty associated with the friction couplings. The method is based on the concept of 'equivalent linear bounds', which assumes that the response is dominated by the input frequency. A frequency-domain description of the system allows the frictional coupling to be modelled using a general describing function, without specifying its functional form. Highlevel constraints are defined that describe known properties of the frictional coupling, which correspond to an admissible region of the describing function. The bounds are found by a combination of numerical optimisation and analytic solutions. The advantage of this approach is that the optimisation only requires calculation of the linear system response and so is very efficient: 
the computational cost of the numerical optimisation is similar to a single Harmonic Balance Method computation.

The method was validated by comparison with numerical and experimental results from an idealised test system. The experimental test rig consisted of an array of eight beams coupled by pin-contact friction dampers. The linear dynamics of the individual beams was characterised by experimental modal analysis, which provided the basis for the numerical benchmark model. The Harmonic Balance Method with just the fundamental retained was used as a reference model in order to provide a 'clean' verification of the bounds method and also extend the range of testing parameters than is possible experimentally. The reference model assumed a Coulomb friction law with a tangential coupling stiffness.

A comparison with an isolated two-beam assembly coupled by one friction damper was tested initially. The response bounds were compared with Monte Carlo HBM results, varying the reference model coefficient of friction and contact stiffness. The bounds exactly encompassed the Monte Carlo results, giving confident verification of the response bounds method. The bounds were then applied to an ensemble of experimental data, which exhibited more limited controlled uncertainty. The bounds again encompassed nearly all the data, but were somewhat conservative for this ensemble of data.

A comparison with the full eight-beam assembly revealed a similar pattern: the numerical Monte Carlo results closely fitted the predicted bounds. For this case, the bounds were also less conservative for the experimental results, as the eight-beam system has more modes within the passband which resulted in greater variability in the response.

There is scope for further investigation: to explore scaling to more complicated systems; to see if it is possible to make the bounds less conservative by including additional information about the frictional nonlinearities; and to include the effect of uncertainties associated with the linear parts of the system (e.g. mistuning). But overall the results are promising: response bounds for an array of friction coupled systems can be computed at similar cost to a single HBM simulation, giving reliable bounds that are valid for both parametric and model uncertainties associated with the friction couplings.

\section{Acknowledgements}

The authors would like to thank Mitsubishi Heavy Industries for funding this research and for granting permission to publish this work. Thanks also to Prof. Robin Langley and Prof. Jim Woodhouse for helpful technical discussions. 


\section{References}

[1] Jim Woodhouse, Thibaut Putelat, and Andrew McKay. Are there reliable constitutive laws for dynamic friction? Philosophical transactions. Series A, Mathematical, physical, and engineering sciences, 373(2051):20140401, 2015.

[2] K. Y. Sanliturk and D. J. Ewins. Modelling Two-Dimensional Friction Contact and Its Application Using Harmonic Balance Method. Journal of Sound and Vibration, 193(2):511-523, jun 1996.

[3] Malte Krack, Sebastian Tatzko, Lars Panning-von Scheidt, and Jörg Wallaschek. Reliability optimization of friction-damped systems using nonlinear modes. Journal of Sound and Vibration, mar 2014.

[4] Benjamin Peherstorfer, Tiangang Cui, Youssef Marzouk, and Karen Willcox. Multifidelity importance sampling. Computer Methods in Applied Mechanics and Engineering, 300:490-509, 2016.

[5] Brian R Mace, Keith Worden, and Graeme Manson. Uncertainty in structural dynamics. Journal of Sound and Vibration, 288:423-429, 2005.

[6] Brian R. MacE, D. V H Vandepitte, and Pascal Lardeur. Uncertainty in structural dynamics. Finite Elements in Analysis and Design, 47(1):1-3, 2011.

[7] David Moens. Uncertainties in structural dynamics. Mechanical Systems and Signal Processing, 32:1-4, 2012.

[8] David Moens and Michael Hanss. Non-probabilistic finite element analysis for parametric uncertainty treatment in applied mechanics: Recent advances. Finite Elements in Analysis and Design, 47(1):4-16, 2011.

[9] N. Wiener. The homogeneous chaos. Amer. J. Math, 60897(4):936, 1938.

[10] George Stefanou. The stochastic finite element method: Past, present and future. Computer Methods in Applied Mechanics and Engineering, 198(912):1031-1051, 2009.

[11] Mohammad Hadigol and Alireza Doostan. Least squares polynomial chaos expansion: A review of sampling strategies. Computer Methods in Applied Mechanics and Engineering, 332:382-407, 2018.

[12] J. Didier, J. J. Sinou, and B. Faverjon. Nonlinear vibrations of a mechanical system with non-regular nonlinearities and uncertainties. Communications in Nonlinear Science and Numerical Simulation, 18(11):3250-3270, 2013.

[13] T. Roncen, J. J. Sinou, and J. P. Lambelin. Experiments and numerical simulations of nonlinear vibrations of a beam with non-ideal boundary conditions and uncertainties. Procedia Engineering, 199:808-813, 2017.

[14] Alfonso M Panunzio and Christoph Schwingshackl. Gt2015-43560 the Study of Stochastic Non-Linear Normal Modes. pages 1-8, 2015. 
[15] V. Cotoni, R. S. Langley, and M. R F Kidner. Numerical and experimental validation of variance prediction in the statistical energy analysis of built-up systems. Journal of Sound and Vibration, 288(3):701-728, 2005.

[16] G. M. Spelman and R. S. Langley. Statistical energy analysis of nonlinear vibrating systems. Philosophical Transactions of the Royal Society A: Mathematical, Physical and Engineering Sciences, 373, 2015.

[17] T. Butlin. Anti-optimisation for modelling the vibration of locally nonlinear structures: An exploratory study. Journal of Sound and Vibration, 332:70997122 , jul 2013.

[18] T. Butlin and A. Batailly. Anti-optimisation applied to the analysis of rotor / stator interaction. In Proceedings of ASME Turbo Expo 2013: Turbine Technical Conference and Exposition, San Antonio, 2013.

[19] T. Butlin. A comparison of worst-case predictions with experimental results for a locally nonlinear beam. Proceedings of ISMA2014 International Conference on Noise and Vibration Engineering (in press), pages 2989-3004, 2014.

[20] T. Butlin. Response bounds for complex systems with a localised and uncertain nonlinearity. Journal of Sound and Vibration, 384:227-252, 2016.

[21] E. P. Petrov and D. J. Ewins. Analytical Formulation of Friction Interface Elements for Analysis of Nonlinear Multi-Harmonic Vibrations of Bladed Disks. Journal of Turbomachinery, 125(2):364, 2003.

[22] Chiara Gastaldi and Teresa M. Berruti. A method to solve the efficiencyaccuracy trade-off of multi-harmonic balance calculation of structures with friction contacts. International Journal of Non-Linear Mechanics, 92(May 2016):25-40, 2017.

[23] K. Y. Sanliturk, M. Imregun, and D. J. Ewins. Harmonic Balance Vibration Analysis of Turbine Blades With Friction Dampers. Journal of Vibration and Acoustics, 119(1):96, 1997.

[24] T. Butlin and J. Woodhouse. Friction-induced vibration: Model development and comparison with large-scale experimental tests. Journal of Sound and Vibration, 332(21):5302-5321, 2013.

[25] D. J. Ewins. Modal testing: theory and practice. Research Studies Press, Ltd., 1986.

[26] A. Cabboi, T. Putelat, and J. Woodhouse. The frequency response of dynamic friction: Enhanced rate-and-state models. Journal of the Mechanics and Physics of Solids, 92:210-236, jul 2016. 